\title{
Modelling and Solving the Combined Inventory Routing Problem with Risk Consideration
}

\author{
Ahmed Kheiri, Konstantinos Zografos \\ Centre for Transport and Logistics \\ Lancaster University Management School, \\ Department of Management Science, \\ Lancaster, LA1 4YX, United Kingdom \\ \{a.kheiri, k.zografos\}@lancaster.ac.uk
}

\begin{abstract}
This work proposes a multi-objective extension of a real-world inventory routing problem (IRP), a generalisation of the classical vehicle routing problem (VRP) with vendor managed inventory (VMI) replenishment. While many mathematical formulations and solution models already exist, this study incorporates businessrelated and risk considerations that makes it unique. It is known that a significant volume of hazardous materials travels every day. Consideration of risks arising from the transportation of hazardous materials as a criterion for selecting distribution routes could potentially reduce the likelihood of accidents and/or the expected consequences of accidents.
\end{abstract}

\section{CCS CONCEPTS}

- Computing methodologies $\rightarrow$ Discrete space search; • Applied computing $\rightarrow$ Transportation;

\section{KEYWORDS}

Inventory routing problem, Hyper-heuristic, Multi-objective optimisation, Risk consideration

\section{ACM Reference Format:}

Ahmed Kheiri, Konstantinos Zografos. 2019. Modelling and Solving the Combined Inventory Routing Problem with Risk Consideration. In Genetic and Evolutionary Computation Conference Companion (GECCO '19 Companion), July 13-17, 2019, Prague, Czech Republic. ACM, New York, NY, USA, 2 pages. https://doi.org/10.1145/3319619.3326773

\section{INTRODUCTION}

Inventory routing problem is a widely studied combinatorial optimisation problem [2]. In this work, we define a multi-objective model to capture the trade-off between risk and cost per delivered unit. The traditional multi-objective shortest path problem belongs to the class of $\mathcal{N} \mathcal{P}$-complete problems identified by Warburton [8], hence the general form multi-objective IRP is also being $\mathcal{N} \mathcal{P}$. complete. Additionally, the average size of the considered problem instances is bigger than those generally studied in the scientific literature.

Permission to make digital or hard copies of part or all of this work for personal or classroom use is granted without fee provided that copies are not made or distributed for profit or commercial advantage and that copies bear this notice and the full citation on the first page. Copyrights for third-party components of this work must be honored.

For all other uses, contact the owner/author(s).

GECCO '19 Companion, July 13-17, 2019, Prague, Czech Republic

(C) 2019 Copyright held by the owner/author(s).

ACM ISBN 978-1-4503-6748-6/19/07 .\$15.00

https://doi.org/10.1145/3319619.3326773

\section{PROBLEM DESCRIPTION}

The detailed description of the inventory routing problem studied in this work can be found in [1]; however, for completeness we also summarise it in this section.

The problem consists in planning the bulk distribution shifts in order to minimise the ratio between the total distribution cost to the total quantity delivered in the long term (also known as logistic ratio) while taking into account risk consideration. We define three types of sites (Base, Customer 'for deliveries' and Source 'for pickups') and two types of resources (Driver and Trailer). A shift is a chronological list of visits made by a vehicle (combination of a driver and a trailer) that must start from the base and end by returning to the same base. Each visit in a shift is defined by a site, product quantity and arrival date. Customers can be VMI customers where sufficient inventory of products is maintained or call-in customers where products are supplied on an on-demand policy. A solution to the problem should avoid stock-outs at the VMI customers and satisfying orders of the call-in customers. We also define a fixed idle time interval in a shift, referred to as layover, to the driver to travel for an extended duration, covering huge geographic areas.

A feasible solution to the inventory routing problem studied in this work must respect the following constraints: Each shift must be assigned to a vehicle (i.e. a driver and a trailer). A vehicle has to start/return from/to the base. Allowed drivers to drive a specific trailer must be respected. Allowed trailers to enter a specific customer site must be respected. Allowed trailers to enter a specific source site must be respected. The capacity of each trailer must be respected. For each VMI customer, sufficient inventory of products must be maintained during the horizon. The delivered quantity at a VMI customer must not exceed the tank capacity. The delivered quantity at a VMI customer must be greater than a predefined quantity. Orders of the call-in customers must be satisfied by at least one operation within the time window. The delivered quantity of a call-in customer must not exceed the ordered quantity. The delivered quantity of a call-in customer must be greater than a predefined minimum quantity. Loading and delivery operations must be contained in one of the opening time windows of the site. Loading and delivery operations take a prefixed setup duration. A driver can be used only during one of its time windows. Driving time per shift must not exceed a predefined maximal driving duration. A predefined duration must separate consecutive shifts assigned by the same driver. Shifts performed by a resource must not overlap in time. The quantity of product in a trailer at the beginning of the shift equals the end quantity of the trailer following the previous shift. A shift cannot have more than one layover. A shift must 
have a layover if and only if there is a visit to one or more layover customers. A layover lasts more than a given layover duration plus driving time. Arrival at a site requires travelling time from previous site, and eventually the layover.

\section{OBJECTIVES}

The first objective of the combined routing and scheduling problem is to minimise the logistic ratio $(L R)$ :

$$
L R=\frac{\sum \forall s \in \text { shifts } S C(s)}{T D Q}
$$

where $S C$ is the shift cost which includes distance cost, time cost, and layover cost; and $T D Q$ is the total delivered quantity.

The second objective is to minimise the total potential risk (i.e. avoiding accident black spots). To accomplish this, we distinguish three different matrices for a given problem instance: $T_{i j}$ which defines the travelling time from site $i$ to site $j, D_{i j}$ which defines the distance between locations $i$ and $j$, and $R_{i j}$ which denotes the transportation risk generated on the path from $i$ to $j . R_{i j}$ is a factor of two attributes as identified in [6]: (i) the probability of a hazardous materials accident, and (ii) the expected population exposed to the impacts of an accident on that link.

\section{SOLUTION MODELS}

In this study, we suggest three solution models to evaluate the quality of feasible solutions.

\subsection{Model I}

The quality of feasible solutions are evaluated using a weighted sum of individuation costs which are the cost per delivered unit and the risk cost.

\subsection{Model II}

The second solution model divides the objective function $f$ into two objectives $f_{1}$ (cost per delivered unit) and $f_{2}$ (risk cost) ordered by descending significance. The ranking of solutions is based on a multi-objective lexicographic order: a solution $S$ is considered better than $S^{\prime}$ if and only if, for some $i \in[1,2], f_{i}(S)<f_{i}\left(S^{\prime}\right)$, and $\forall j$ such that $j<i, j \geq 1, f_{j}(S)=f_{j}\left(S^{\prime}\right)$.

\subsection{Model III}

The third solution model uses the goal programming approach which consists in transforming the multi-objective formulation to a single-objective model by setting goals for each objective and minimising the deviation from these goals.

\section{OPTIMISATION METHODS}

Although, the traditional optimisation techniques such as integer programming [7], showed success in solving small sizes of the inventory routing problems; however, such methods cannot be employed when the number of routes increases and when several resources are considered. Instead, researchers have made several efforts to develop efficient heuristic and meta-heuristic methods to generate the routes and schedules. The most frequently preferred meta-heuristic method for inventory routing problem is local search [2]. In this study, we will be performing experiments using hyperheuristics [3].
We propose a sequence-based selection hyper-heuristic utilising a hidden Markov model $[4,5]$. This adaptive approach maintains scores representing the probability of choosing a low level heuristic considering the previously invoked heuristic, learning effective sequences of low level heuristics to employ. The scores are updated using a Reinforcement Learning strategy during the search process. The proposed hyper-heuristic outperforms other competing methods of ROADEF/EURO 2016 Challenge on inventory routing problem against, 12 finalists teams, producing the best solutions across all of the released problem instances. The general framework of the algorithm is presented in Figure 1.

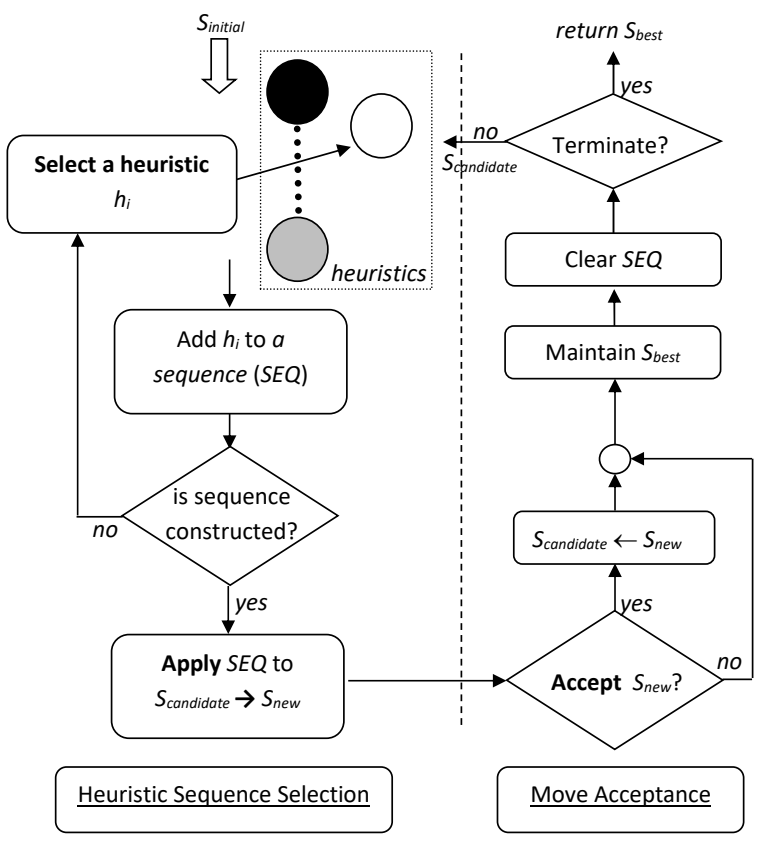

Figure 1: Sequence-based selection hyper-heuristic

\section{REFERENCES}

[1] Jean Andrè, Michele Quattrone, Rodrigue Fokouop, Eric Bourreau, Vincent Jost, Safia Kedad-Sidhoum, David Savourey, and Marc Sevaux. 2016. ROADEF/EURO Challenge 2016: Inventory Routing Problem. http://challenge.roadef.org/2016/en/ index.php. (2016)

[2] Thierry Benoist, Frédéric Gardi, Antoine Jeanjean, and Bertrand Estellon. 2011. Randomized local search for real-Life inventory routing. Transportation Science 45, 3 (2011), 381-398.

[3] E. K. Burke, M. Gendreau, M. Hyde, G. Kendall, G. Ochoa, E. Özcan, and R. Qu. 2013. Hyper-heuristics: a survey of the state of the art. Fournal of the Operational Research Society 64, 12 (2013), 1695-1724.

[4] Ahmed Kheiri and Ed Keedwell. 2015. A sequence-based selection hyper-heuristic utilising a hidden Markov model. In Proceedings of the 2015 on Genetic and Evolutionary Computation Conference (GECCO '15). ACM, New York, NY, USA, 417-424.

[5] Ahmed Kheiri and Ed Keedwell. 2017. A Hidden Markov Model Approach to the Problem of Heuristic Selection in Hyper-Heuristics with a Case Study in High School Timetabling Problems. Evolutionary Computation 25, 3 (2017), 473-501.

[6] George F. List, Pitu B. Mirchandani, Mark A. Turnquist, and Konstantinos G. Zografos. 1991. Modeling and Analysis for Hazardous Materials Transportation: Risk Analysis, Routing/Scheduling and Facility Location. Transportation Science 25, 2 (1991), 100-114.

[7] Oğuz Solyalı and Haldun Süral. 2011. A Branch-and-Cut Algorithm Using a Strong Formulation and an A Priori Tour-Based Heuristic for an Inventory-Routing Problem. Transportation Science 45, 3 (2011), 335-345.

[8] Arthur Warburton. 1987. Approximation of Pareto Optima in multiple-objective, shortest-path problems. Operations Research 35, 1 (1987), 70-79. 